\title{
Pain Distribution and Predictors of Widespread Pain in the Immediate Aftermath of Motor Vehicle Collision
}

Andrey V. Bortsov ${ }^{1}$, Timothy F. Platts-Mills ${ }^{2}$, David A. Peak ${ }^{3}$, Jeffrey S. Jones ${ }^{4}$, Robert A. Swor $^{5}$, Robert M. Domeier ${ }^{6}$, David C. Lee ${ }^{7}$, Niels K. Rathlev ${ }^{8}$, Phyllis L. Hendry ${ }^{9}$, Roger B. Fillingim $^{10}$, and Samuel A. McLean ${ }^{1,2}$

${ }^{1}$ Department of Anesthesiology, University of North Carolina, Chapel Hill, NC, USA

${ }^{2}$ Department of Emergency Medicine, University of North Carolina, Chapel Hill, NC, USA

${ }^{3}$ Department of Emergency Medicine, Massachusetts General Hospital, Boston, MA, USA

${ }^{4}$ Department of Emergency Medicine, Spectrum Health Hospital - Butterworth Campus, Grand Rapids, MI, USA

${ }^{5}$ Department of Emergency Medicine, William Beaumont Hospital, Royal Oak, MI, USA

${ }^{6}$ Department of Emergency Medicine, St Joseph Mercy Hospital, Ann Arbor, MI, USA

${ }^{7}$ Department of Emergency Medicine, North Shore University Hospital, Manhasset, NY, USA

${ }^{8}$ Department of Emergency Medicine, Baystate Medical Center, Springfield, MA, USA

${ }^{9}$ Department of Emergency Medicine, College of Medicine, University of Florida and Shands Jacksonville, Jacksonville, FL, USA

${ }^{10}$ Department of Community Dentistry and Behavioral Science, College of Dentistry, University of Florida, Gainesville, FL, USA

\section{Abstract}

Background-Musculoskeletal pain is common after motor vehicle collision. The study objective was to evaluate distribution of pain and predictors of widespread musculoskeletal pain in the early aftermath (within $48 \mathrm{~h}$ ) of collision.

Methods-European American adults aged 18-65 years presenting to the emergency department after collision who were discharged to home after evaluation were eligible. Evaluation included an assessment of reported pre-collision psychological characteristics, crash characteristics, current pain severity and location, and current psychological symptoms. Adjusted risk ratios were estimated using generalized linear models.

Results-Among 890 participants included in the study, 589/890 (66\%) had pain in three or more regions, and 192/890 (22\%) had widespread musculoskeletal pain (pain in 7 or more regions). In adjusted analyses, the presence of widespread pain was strongly associated with depressive and somatic symptoms prior to collision, pain catastrophizing, and acute psychological

Corresponding author: Samuel A McLean, MD, Department of Anesthesiology, The University of North Carolina at Chapel Hill; Address: N2201 UNC Hospitals CB 7010, Chapel Hill, NC 27599-7010; smclean@aims.unc.edu; Phone: 919.843.5931; Fax: 919.966.7193.

Disclosures: Dr. Fillingim is an officer and stockholder in Algynomics, Inc.

Author contributions

$\mathrm{AB}, \mathrm{TPM}, \mathrm{RF}$, and SM substantially contributed to study conception, design, data analysis and interpretation; DP, JJ, RS, RD, DL, $\mathrm{NR}$, and PH contributed to data acquisition; AB and SM drafted the manuscript; TPM, DP, JJ, RS, RD, DL, NR, PH, and RF critically revised the manuscript for important intellectual content. All authors have approved the final version of the manuscript. 
symptoms, and was not associated with most collision characteristics (road speed limit, extent of vehicle damage, collision type, driver vs. passenger, airbag deployment). The reported number of body regions that struck an object during the collision was associated with both reported precollision depressive symptoms and with widespread pain.

Conclusion-More than one in five individuals presenting to the emergency department in the hours after motor vehicle collision have widespread pain. Widespread pain is strongly associated with patient characteristics known to be modulated by supraspinal mechanisms, suggesting that stress-induced hyperalgesia may influence acute widespread pain after collision.

\section{Introduction}

The great majority of individuals who experience a motor vehicle collision (MVC) do not have a fracture or other acute injury requiring immediate medical intervention or hospitalization (Platts-Mills et al., 2012b; Thygerson et al., 2011). Acute pain among these patients is often ascribed to "musculoskeletal strain". The majority of patients with acute pain recover, but a subset transition to persistent pain (Holm et al., 2008). Persistent musculoskeletal pain after MVC is an important public health problem (Holm et al., 2008), and may occur in a number of body regions (Cassidy et al., 2003; Hincapie et al., 2010) and/ or may be widespread (Holm et al., 2007; Jones et al., 2011; Wynne-Jones et al., 2006).

To date, studies examining acute pain after MVC have focused on the neck region (Berglund et al., 2001; Kasch et al., 2001; Norris and Watt, 1983). A more comprehensive assessment of the distribution and extent of acute pain in the early aftermath of MVC may enrich our understanding of patients' experiences. In addition, evaluating the distribution and extent of acute pain symptoms and the relationship between individual and collision-related characteristics and pain extent may suggest biological mechanisms of acute post-MVC pain pathogenesis.

In this study we evaluated the distribution, extent, and severity of pain symptoms among a large cohort of individuals who presented for emergency evaluation in the hours after MVC and were subsequently discharged to home. We hypothesized that multiple regions of body pain would be the norm, and that a substantial subset of patients would have pain in seven or more body regions ("widespread pain"). We based this hypothesis on preclinical and clinical data suggesting that stress-induced hyperalgesia may be an important biological mechanism contributing to acute pain after stressful events such as MVC (McLean, 2011). Such stressinduced hyperalgesia is relatively widespread in nature (Imbe et al., 2006; Khasar et al., 2003; Vidal and Jacob, 1982), and thus might be expected to contribute to acute pain across a number of body regions.

We also assessed sociodemographic, health-related, crash-related, and psychological factors associated with acute post-MVC widespread pain. We hypothesized that widespread pain would be strongly associated with acute psychological symptoms. We based this hypothesis on data indicating that acute psychological responses are modulated by central neurobiological systems (Pervanidou and Chrousos, 2010) which also appear to be involved in stress-induced hyperalgesia (Imbe et al., 2006). Because we hypothesized that biological mechanisms contributing to acute post-MVC widespread pain are strongly influenced by stress-induced mechanisms which are independent of tissue injury, we hypothesized that collision-related characteristics would be less strongly associated with the presence of widespread pain than psychological measures. 


\section{Methods}

\subsection{Study participants}

Participants in this study were individuals aged 18 to 65 years who presented to one of eight emergency departments (EDs) for care after MVC. Only individuals who spoke English and who were alert, oriented, and clinically stable were enrolled. In addition, we excluded patients who had spinal, facial, long bone, and skull fractures as well as prisoners, pregnant patients, patients with intracranial injury or laceration with significant hemorrhage, patients who were admitted to the hospital, and patients presenting to the ED more than 24 hours after MVC. In addition, to reduce sample heterogeneity, patients who rode a motorcycle or scooter at the time of collision were excluded. The study was approved by the Institutional Review Board at each study site (Bay State Medical Center, Springfield, MA; Beaumont Hospital, Troy, Michigan, USA; Beaumont Hospital, Royal Oak, Michigan, USA; Massachusetts General Hospital, Boston, Massachusetts, USA; North Shore University Hospital, Manhasset, New York, USA; Saint Joseph Mercy Health System, Ann Arbor, Michigan, USA; Spectrum Health, Grand Rapids, Michigan, USA; Shands Jacksonville, Jacksonville, Florida, USA). Participants were recruited as part of an ongoing R01 study (AR056328). Because other portions of the study will include genetic analyses, only patients who identified themselves as European American were recruited in order to reduce population stratification bias (Diatchenko et al., 2007). All participants gave written informed consent.

\subsection{Data collection procedures}

A research assistant stationed in each of the study site EDs monitored the chief complaint and injury history of presenting patients. Potentially eligible patients were approached to determine eligibility and eligible patients were offered enrollment into the study. The research assistant informed patients of the voluntary nature of their participation, discussed the risks and benefits of the study, and informed patients that their participation could be withdrawn at any time. In addition, in order to assess patient competency to give informed consent, patients were required to describe the essential elements of the study back to the research assistant. Following consent, study participants completed an ED interview using a web-based survey with explicit definitions of variables. Before enrolling patients in the ED, each research assistant completed a study training module followed by an interview with a standardized mock ED patient. Comparison of mock ED patient data across research assistants demonstrated high concordance, with an error rate of only $1.3 \%$. To enhance participation and to compensate participants for their time during their ED visit, study participants were provided with $\$ 80.00$ remuneration for completing the ED interview. After study participant discharge from the ED, a research assistant completed a web-based data extraction form. This form collected information from ED and hospital medical records.

\subsection{Measures}

Demographic information was assessed using standardized questionnaire items. Pain symptom location was evaluated using the Regional Pain Scale (Wolfe, 2003). This scale has been shown to be a valid and reliable measure of pain location and distribution (Wolfe, 2003). Current pain intensity in each body region and current overall pain severity were assessed via a numeric rating scale (NRS) ranging from 0 ("no pain") to 10 ("worst pain possible"). If participants reported pain they were also asked whether the pain was related to the MVC; only MVC-related pain (greater than $99 \%$ of all pain reported) was included in the present analyses. Peritraumatic distress was assessed using the Peritraumatic Distress Inventory (PDI). The PDI is a reliable and valid instrument for measuring distress during the peritraumatic period (Brunet et al., 2001; Udelsman et al., 1987). Total scores can range from 0 to 52, with scores greater than 23 indicating severe distress (Nishi et al., 2010). 
Perceived threat to life was assessed using 0-10 numeric scale. Information regarding the collision was assessed using a structured questionnaire developed in consultation with biomechanics experts at the University of Michigan Transportation Research Institute. This questionnaire included questions regarding position inside the vehicle (driver or passenger), airbag deployment, number of body regions striking the vehicle interior or other object during the collision, road speed limit, location of primary vehicle damage, and extent of vehicle damage (none, minor, moderate, severe/car not drivable). Information regarding collision characteristics assessed using this interview have been shown to demonstrate excellent agreement with collision information recorded by police at the scene of the MVC (Lee et al., 2012). Patient injuries were scored using the Abbreviated Injury Scale (Baker et al., 1974).

Beliefs regarding emotional recovery were assessed by asking participants to estimate their expected time to emotional recovery (Carosella et al., 1994). Pain catastrophizing was assessed using the Pain Catastrophizing Scale (Sullivan et al., 1995). Pain Catastrophizing Scale scores range from $0-52$, with scores of 30 or more indicating an increased propensity to catastrophize (Sullivan et al., 1995). Dissociation was assessed using the Michigan Critical Events Perception Scale (MCEPS) (Michaels et al., 1999). MCEPS scores range from 5-25, with scores of 15 or more indicating significant dissociation (Michaels et al., 1999). After assessment of current pain and psychological symptoms, participants were also asked about pain symptoms during the previous calendar month. For the assessment of past pain, the Regional Pain Scale (Wolfe, 2003) and numeric rating scale assessments were again used. The presence and severity of 21 common somatic symptoms during the calendar month prior to MVC was also assessed (Adapted from Pennebaker, 1982); such symptoms have been shown to predict chronic pain (McBeth et al., 2005). The Center for Epidemiological Studies Depression (CESD) Scale, a 20-item self-report questionnaire, was used to assesses symptoms of depression (Radloff, 1977) during the calendar month prior to MVC. CESD scores range from 0 to 60 , with scores of 26 or more indicating substantial depressive symptoms (Geisser et al., 1997). SF-12 physical component summary and mental component summary scores were used to evaluate general physical and mental health during the month prior to the MVC (Ware et al., 1996). Transformed scale scores for these measures range from 0 to 100 , with higher scores indicating better mental or physical health (Ware et al., 1996). Additional details regarding the study design and measurements have been published (Platts-Mills et al., 2011).

\subsection{Statistical analysis}

The number of body regions with acute pain was calculated by summing the number of body regions in which participants reported any MVC-related pain at the time of ED assessment (defined as NRS score $>0$ ). Widespread pain was defined by the presence of seven or more body regions with pain (Wolfe et al., 2010). When evaluating the association between candidate predictors and widespread pain, predictors measured on a continuous scale were categorized to facilitate effect size assessment and to account for potential non-linear effects. Predictors were categorized using cut-off scores demonstrated in the literature to define clinically relevant categories. In the event that such cut-off scores were not available, tertiles were used. Relative risks and confidence intervals for associations were estimated using generalized linear models for binary outcomes after adjustment for potential confounders. Associations between sociodemographic characteristics and the presence of widespread pain were adjusted for study site. Associations between crash-related characteristics and the presence of widespread pain were adjusted for age, sex, education, and study site. Associations between pre-crash health and psychological factors and the presence of widespread pain were adjusted for age, sex, education, study site, and the number of body parts striking an object in the vehicle during the collision. All statistical analyses were 
performed using SAS (version 9.2, SAS Institute Inc., Cary, NC). P-values $<0.05$ were considered statistically significant.

\section{Results}

\subsection{Sample acquisition and socio-demographic characteristics}

A total of 10,629 patients were screened, 1,416 were eligible, 969 consented to study participation, and 948 completed baseline evaluation (Figure 1). After exclusion of those whose collision occurred while driving a motorcycle or a scooter $(n=58), 890$ study participants were available for analyses. Most study participants were female, less than 40 years of age, and did not have a college degree. The median time between MVC and ED presentation was 1 hour and 14 minutes. Three quarters of study participants presented to the ED within 2.5 hours of the MVC. Nearly three quarters $(634 / 890,71 \%)$ of study participants came to the ED directly from the scene of the collision, and more than half $(493 / 890,56 \%)$ were transported to the ED by ambulance. Patients had minor injury as classified by anatomical scoring systems. Ninety nine percent of participants had an Abbreviated Injury Scale score of one, the remaining one percent had a score of two.

\subsection{Number of body regions with pain in the hours after MVC}

Nearly all study participants $(827 / 890,93 \%)$ had some MVC-related pain; two thirds $(589 / 890,66 \%)$ had pain in three or more regions (Figure 2). Widespread pain (pain in seven or more body regions) was present in more than one in five participants (192/890, 22\%). Moderate or severe neck pain (NRS $>3$ ) was present in more than half of the study participants (488/890,55\%); and 161/192 (84\%) of patients with widespread pain also had moderate or severe neck pain. The number of body regions with pain was moderately correlated with increased overall pain severity (Pearson $r=0.38, p<0.001$, Figure 2 ) and with neck pain severity $(r=0.13, p=0.001)$. Body regions with the highest prevalence of pain in the early aftermath of MVC were the neck, head, upper back, lower back, and shoulders (Figure 3).

\subsection{Association between socio-demographic characteristics and the risk of widespread pain in the hours after MVC}

Associations between socio-demographic characteristics and the risk of widespread pain in the hours after minor MVC, adjusted for participant study site, are shown in Table 1. Women had a higher risk of widespread pain than men (Table 1). Participants with some post-high school or college education had higher risk of widespread pain than those with higher or lower education levels (Table 1).

\subsection{Association between crash characteristics and widespread pain in the hours after MVC}

Associations between collision-related characteristics and the presence of widespread pain in the acute aftermath of MVC, adjusted for age, sex, education, and study site, are shown in Table 1. Vehicle characteristics selected as markers of crash severity/collision energy were not associated with the presence of widespread pain. An individual injury characteristic, the number of participant body regions which struck an object during the collision, was associated with the presence of acute post-MVC widespread pain (adjusted relative risk of 1.51 (95\% CI 1.05 to 2.18) for striking one body region and 2.38 (95\% CI 1.67 to 3.39 ) for striking multiple body regions). Of note, the number of body regions striking the vehicle was associated with both a non-rear end collision $(p<.0001)$ and increased reported depressive symptoms during the month prior to collision $(\mathrm{r}=.13, \mathrm{p}=.0002)$ and increased reported somatic symptoms reported during the month prior to collision $(r=.08, \mathrm{p}=.02)$. Striking an object during collision was not a necessary antecedent to the presence of acute 
widespread pain: $37 / 267(14 \%)$ of those who did not strike a body part reported widespread pain, including $9 / 60(15 \%)$ of those who had only minor vehicle damage.

\subsection{Association between health and psychological factors and the presence of widespread pain in the hours after MVC}

After adjustment for study site, socio-demographic characteristics, and the number of body parts striking an object during the collision, a greater number of somatic symptoms in the calendar month prior to collision was strongly associated with the presence of widespread pain in the acute aftermath of MVC. Increased depressive symptoms and the number of body regions with pain during the calendar month prior to collision were also significantly associated with widespread pain. Pain catastrophizing also strongly associated with widespread pain; this strong association remained after adjustment for overall pain severity at the time of pain catastrophizing assessment. There was no association between mental and physical health subscales of SF-12 and the presence of widespread pain in the acute aftermath of MVC.

\subsection{Association between immediate post-MVC psychological outcomes and the presence of widespread pain in the hours after MVC}

Associations between immediate post-MVC psychological responses and the presence of widespread pain were evaluated adjusting for sociodemographic factors, collision-related factors, and study site. All of the psychological responses to MVC assessed (perceived life threat, peritraumatic distress, dissociation symptoms, and estimated time to emotional recovery) were strongly associated with the presence of widespread pain (Table 2).

\section{Discussion}

In this observational study of adult European Americans evaluated in the ED in the hours after MVC and subsequently discharged to home, pain in 3 or more body regions was the norm, and more than one in five participants experienced pain in 7 or more body regions (defined as "widespread pain"). The body regions with the highest prevalence of acute pain after MVC were the neck, head, upper back, lower back, and shoulders. Consistent with our study hypothesis that biological mechanisms contributing to acute post-MVC widespread pain are strongly influenced by stress-induced mechanisms which are independent of tissue injury, the presence of widespread pain was strongly associated with depressive and somatic symptoms prior to collision, pain catastrophizing, and acute psychological symptoms in response to trauma, and generally not associated with crash-related characteristics.

There was one exception to this general lack of association between crash-related characteristics and the presence of widespread pain: the number of body regions that participants reported struck an object during the collision was strongly associated with the presence of widespread pain. In bivariate analyses, this characteristic was strongly associated with collision direction, with a greater number of body regions struck in front or side impact collisions. This bivariate association is consistent with tissue injury biomechanics, as the great majority of our study participants (86\%) were drivers, and collisions from the front or side are higher energy collisions (Bedard et al., 2002) which are more likely to result in contact with the driver's side door, steering wheel, and/or dash board. Interestingly, in bivariate analyses participant recollection of the number of body regions struck was also associated with depressive and somatic symptom burden prior to collision. We hypothesize that number of body regions reported struck was associated with widespread pain both because it is the most direct, accurate measure of the contribution of injury severity to widespread pain, and also because this report was influenced by 
psychological characteristics which have shared vulnerability with experiencing stressinduced hyperalgesia.

We based our study hypothesis on preclinical and clinical data suggesting that stressinduced hyperalgesia, which is relatively widespread in nature (Imbe et al., 2006; Khasar et al., 2003; Vidal and Jacob, 1982), contributes to acute pain after stressful events such as MVC (McLean, 2011). Considered together, our study findings support this hypothesis: (i) acute post-MVC pain was commonly present in a number of body regions; (ii) pain in 7 or more body regions (widespread pain) was present in more than one in five individuals; (iii) individual characteristics modulated by central neurobiological systems involved in stressinduced hyperalgesia (increased depressive and somatic symptoms prior to MVC, pain catastrophizing, and acute psychological responses to collision) were strongly associated with the presence of widespread pain, and (iv) widespread pain occurred in $14 \%$ of the participants with no body areas struck during collision (vs. 22\% of all patients), and in $18 \%$ of participants with no or minor vehicle damage.

Previous studies indicate that some individuals develop chronic widespread pain after the types of MVCs included in this study (Buskila et al., 1997; Holm et al., 2007; Wynne-Jones et al., 2006). In a prospective study of Canadians filing MVC-related insurance claims assessed 6 weeks and 4, 6, and 12 months after MVC, Holm et al found widespread pain to be present in $21 \%$ of individuals at one or more time points. Among individuals experiencing widespread pain, more than half had developed widespread pain prior to 6 week evaluation (Holm et al., 2007). Our study results extend these findings and suggest that for some individuals experiencing MVC, widespread pain may develop within hours, rather than developing initially as regional pain and then gradually transitioning to widespread over a period of weeks or months. This hypothesis is supported by the consistency of predictors for acute widespread pain identified in this study and predictors of chronic widespread pain identified in previous studies (Holm et al., 2007; Wynne-Jones et al., 2006).

Several limitations should be considered when interpreting our study results. First, our study was limited to European Americans age 18 to 65 years of age who presented to the ED for evaluation. The extent of pain following MVC and determinants of widespread pain may differ in other ethnic and racial groups, older adults (Platts-Mills et al., 2012a), and individuals who do not come to the emergency department for evaluation after MVC. In addition, our study focused on individuals who were evaluated in the ED and subsequently discharged to home. The extent of pain and determinants of widespread pain may differ among those experiencing more serious injuries who are admitted to the hospital, however in the United States more than $90 \%$ of all individuals who present to the ED for evaluation after MVC are discharged to home after evaluation (Platts-Mills et al., 2012b). Finally, widespread pain present prior to MVC might have biased our results. However, widespread pain in the month prior to MVC was reported by only $3 \%$ of participants, and only $6 \%(\mathrm{n}=$ 11) of those who developed widespread pain after MVC $(n=192)$ reported widespread pain during the calendar month prior to MVC. In addition, in our analyses we used only pain that participants reported as MVC-related, and none of our participants was engaged in litigation. Given these considerations, we believe that pre-existing widespread pain is unlikely to bias substantially our study results.

In summary, our findings indicate that widespread pain in the early aftermath of MVC is common. The onset of widespread pain within hours of MVC and the association of widespread pain with factors known to be modulated by supraspinal mechanisms suggest that stress-induced hyperalgesia may contribute to the development of acute widespread pain. Further studies examining the etiology and outcomes of acute post-MVC widespread 
pain are needed, and may lead to new methods to prevent and treat widespread pain developing after MVC.

\section{Acknowledgments}

Funding sources: Funding for this study was provided by the National Institute of Arthritis, Musculoskeletal, and Skin Diseases (R01 AR056328: Samuel A. McLean). Dr. Platts-Mills is supported by Award Number KL2 RR025746 from the National Center for Research Resources.

\section{References}

Baker SP, O’Neill B, Haddon W, Long WB. The injury severity score: a method for describing patients with multiple injuries and evaluating emergency care. J Trauma. 1974; 14:187-196. [PubMed: 4814394]

Bedard M, Guyatt GH, Stones MJ, Hirdes JP. The independent contribution of driver, crash, and vehicle characteristics to driver fatalities. Accid Anal Prev. 2002; 34:717-727. [PubMed: 12371777]

Berglund A, Alfredsson L, Jensen I, Cassidy JD, Nygren A. The association between exposure to a rear-end collision and future health complaints. J Clin Epidemiol. 2001; 54:851-856. [PubMed: 11470396]

Brunet A, Weiss DS, Metzler TJ, Best SR, Neylan TC, Rogers C, Fagan J, Marmar CR. The Peritraumatic Distress Inventory: a proposed measure of PTSD criterion A2. Am J Psychiatry. 2001; 158:1480-1485. [PubMed: 11532735]

Buskila D, Neumann L, Vaisberg G, Alkalay D, Wolfe F. Increased rates of fibromyalgia following cervical spine injury. A controlled study of 161 cases of traumatic injury. Arthritis Rheum. 1997; 40:446-452. [PubMed: 9082932]

Carosella AM, Lackner JM, Feuerstein M. Factors associated with early discharge from a multidisciplinary work rehabilitation program for chronic low back pain. Pain. 1994; 57:69-76. [PubMed: 8065799]

Cassidy JD, Carroll L, Cote P, Berglund A, Nygren A. Low back pain after traffic collisions: a population-based cohort study. Spine. 2003; 28:1002-1009. [PubMed: 12768138]

Diatchenko L, Nackley AG, Slade GD, Belfer I, Max MB, Goldman D, Maixner W. Responses to Drs. Kim and Dionne regarding comments on Diatchenko, et al. Catechol-O-methyltransferase gene polymorphisms are associated with multiple pain-evoking stimuli. Pain. 2007; 129:366-370. [PubMed: 17851590]

Geisser ME, Roth RS, Robinson ME. Assessing depression among persons with chronic pain using the Center for Epidemiological Studies-Depression Scale and the Beck Depression Inventory: a comparative analysis. Clin J Pain. 1997; 13:163-170. [PubMed: 9186024]

Hincapie CA, Cassidy JD, Cote P, Carroll LJ, Guzman J. Whiplash injury is more than neck pain: a population-based study of pain localization after traffic injury. J Occup Environ Med. 2010; 52:434-440. [PubMed: 20357684]

Holm LW, Carroll LJ, Cassidy JD, Hogg-Johnson S, Cote P, Guzman J, Peloso P, Nordin M, Hurwitz E, van der Velde G, et al. The burden and determinants of neck pain in whiplash-associated disorders after traffic collisions: results of the Bone and Joint Decade 2000-2010 Task Force on Neck Pain and Its Associated Disorders. Spine. 2008; 33:S52-59. [PubMed: 18204401]

Holm LW, Carroll LJ, Cassidy JD, Skillgate E, Ahlbom A. Widespread pain following whiplashassociated disorders: incidence, course, and risk factors. J Rheumatol. 2007; 34:193-200. [PubMed: 17143964]

Imbe H, Iwai-Liao Y, Senba E. Stress-induced hyperalgesia: animal models and putative mechanisms. Front Biosci. 2006; 11:2179-2192. [PubMed: 16720304]

Jones GT, Nicholl BI, McBeth J, Davies KA, Morriss RK, Dickens C, Macfarlane GJ. Role of road traffic accidents and other traumatic events in the onset of chronic widespread pain: Results from a population-based prospective study. Arthritis Care Res. 2011; 63:696-701. 
Kasch H, Stengaard-Pedersen K, Arendt-Nielsen L, Staehelin Jensen T. Headache, neck pain, and neck mobility after acute whiplash injury: a prospective study. Spine. 2001; 26:1246-1251. [PubMed: 11389391]

Khasar SG, Green PG, Miao FJ, Levine JD. Vagal modulation of nociception is mediated by adrenomedullary epinephrine in the rat. Eur J Neurosci. 2003; 17:909-915. [PubMed: 12603283]

Lee YM, Platts-Mills TF, MacWilliams JB, Sochor MR, Jones JS, Domeier RM, Schneider LW, McLean SA. Descriptions of Motor Vehicle Collisions by Participants in Emergency DepartmentBased Studies: Are they Accurate? West J Emerg Med. 2012; 13:329-34. [PubMed: 22942933]

McBeth J, Silman AJ, Gupta A, Chiu YH, Ray D, Morriss R, Dickens C, King Y, Macfarlane GJ. Moderation of psychosocial risk factors through dysfunction of the hypothalamic-pituitary-adrenal stress axis in the onset of chronic widespread musculoskeletal pain: findings of a population-based prospective cohort study. Arthritis Rheum. 2007; 56:360-71. [PubMed: 17195240]

McLean SA. The potential contribution of stress systems to the transition to chronic whiplashassociated disorders. Spine. 2011; 36:S226-232. [PubMed: 22020617]

Michaels AJ, Michaels CE, Zimmerman MA, Smith JS, Moon CH, Peterson C. Posttraumatic stress disorder in injured adults: etiology by path analysis. J Trauma. 1999; 47:867-873. [PubMed: 10568714]

Nishi D, Matsuoka Y, Yonemoto N, Noguchi H, Kim Y, Kanba S. Peritraumatic Distress Inventory as a predictor of post-traumatic stress disorder after a severe motor vehicle accident. Psychiatry Clin Neurosci. 2010; 64:149-156. [PubMed: 20447011]

Norris SH, Watt I. The prognosis of neck injuries resulting from rear-end vehicle collisions. J Bone Joint Surg Br. 1983; 65:608-611. [PubMed: 6643566]

Pennebaker, JW.; Watson, D. Current Concepts of Somatization: Research and Clinical Perspectives. 1991. The Psychology of Somatic Symptoms; p. 21

Pervanidou P, Chrousos GP. Neuroendocrinology of post-traumatic stress disorder. Prog Brain Res. 2010; 182:149-160. [PubMed: 20541663]

Platts-Mills TF, Ballina L, Bortsov AV, Soward A, Swor RA, Jones JS, Lee DC, Peak DA, Domeier RM, Rathlev NK, et al. Using emergency department-based inception cohorts to determine genetic characteristics associated with long term patient outcomes after motor vehicle collision: methodology of the CRASH study. BMC Emerg Med. 2011; 11:14. [PubMed: 21943293]

Platts-Mills TF, Burke GF, Lee YM, Swor RA, Zaleski EZ, Clauw DJ, McLean SA. Pain and interference of pain with function and mood in elderly adults involved in a motor vehicle collision: a pilot study. Exp Aging Res. 2012a; 38:330-343. [PubMed: 22540386]

Platts-Mills TF, Hunold KM, Esserman DA, Sloane PD, McLean SA. Motor Vehicle Collision-related Emergency Department Visits by Older Adults in the United States. Acad Emerg Med. 2012b; 19:821-827. [PubMed: 22724382]

Radloff LS. The CES-D Scale: A self-report depression scale for research in the general population. Applied Psychological Measurement. 1977; 1:385-401.

Sullivan MJ, Bishop S, Pivik J. The Pain Catastrophizing Scale: development and validation. Psychological Assessment. 1995; 7:524-532.

Thygerson SM, Merrill RM, Cook LJ, Thomas AM, Wu AC. Epidemiology of motor vehicle crashes in Utah. Traffic Inj Prev. 2011; 12:39-47. [PubMed: 21259172]

Udelsman R, Norton JA, Jelenich SE, Goldstein DS, Linehan WM, Loriaux DL, Chrousos GP. Responses of the hypothalamic-pituitary-adrenal and renin-angiotensin axes and the sympathetic system during controlled surgical and anesthetic stress. J Clin Endocrinol Metab. 1987; 64:986994. [PubMed: 3031124]

Vidal C, Jacob J. Hyperalgesia induced by non-noxious stress in the rat. Neurosci Lett. 1982; 32:7580. [PubMed: 7145227]

Ware JE, Kosinski M, Keller SD. A 12-item short-form health survey. Med Care. 1996; 34:220-233. [PubMed: 8628042]

Wolfe F. Pain extent and diagnosis: development and validation of the regional pain scale in 12,799 patients with rheumatic disease. J Rheumatol. 2003; 30:369-378. [PubMed: 12563698] 
Wolfe F, Clauw DJ, Fitzcharles MA, Goldenberg DL, Katz RS, Mease P, Russell AS, Russell IJ, Winfield JB, Yunus MB. The American College of Rheumatology preliminary diagnostic criteria for fibromyalgia and measurement of symptom severity. Arthritis Care Res. 2010; 62:600-610.

Wynne-Jones G, Jones GT, Wiles NJ, Silman AJ, Macfarlane GJ. Predicting new onset of widespread pain following a motor vehicle collision. J Rheumatol. 2006; 33:968-974. [PubMed: 16541476] 


\section{What's already known about this topic?}

- Persistent musculoskeletal pain after motor vehicle collision (MVC) is an important public health problem.

- Some individuals may develop persistent widespread pain after MVC.

\section{What does this study add?}

- A substantial number of individuals experiencing minor motor vehicle collision develop widespread musculoskeletal pain in the early aftermath (within 48 hours) of MVC.

- Widespread pain in these individuals is associated with pre-crash characteristics known to be modulated by supraspinal mechanisms. 


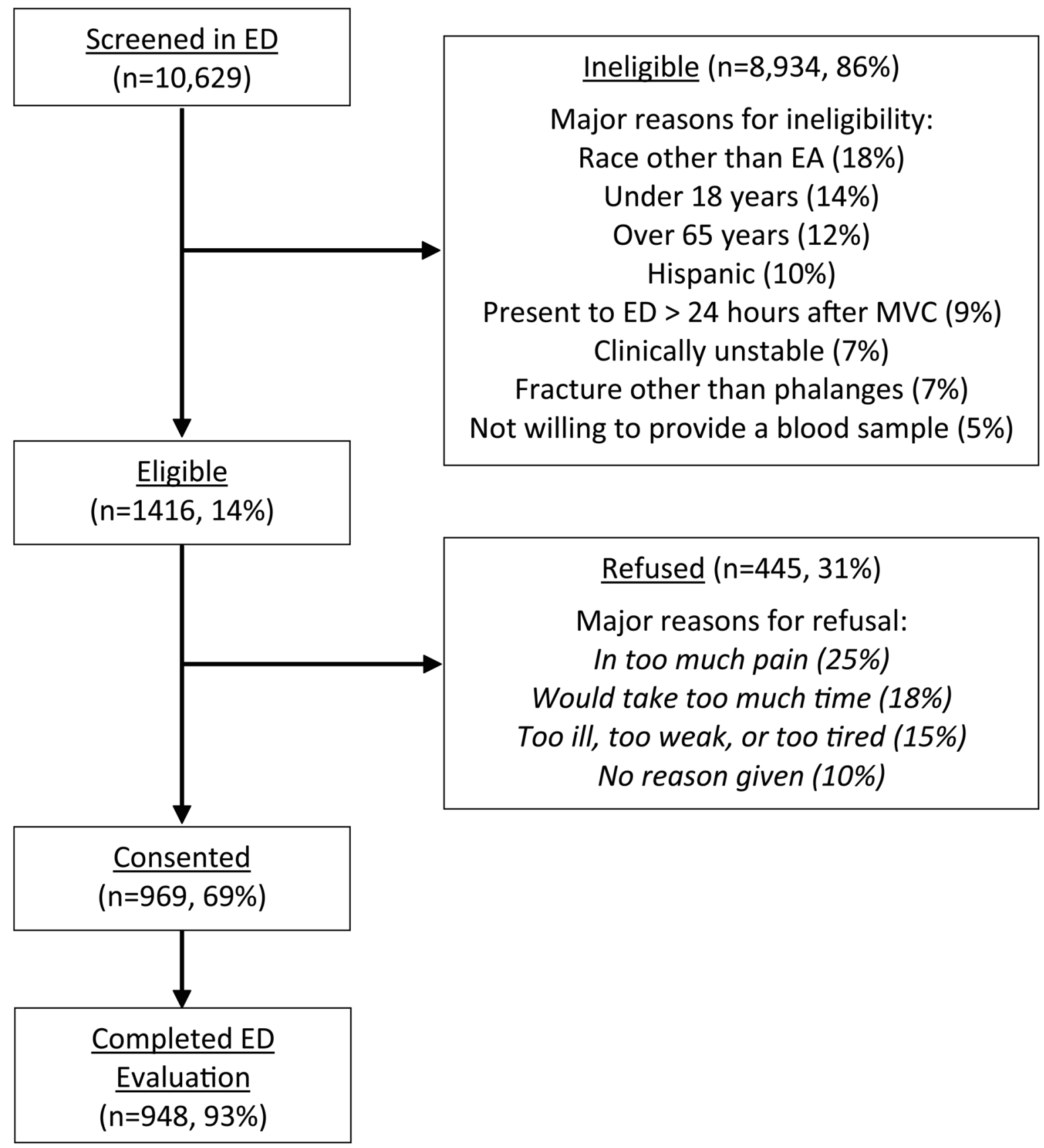

Figure 1. Study recruitment flowchart

Patients may have multiple reasons for ineligibility or refusal. ED, Emergency Department;

EA, European American; MVC, motor vehicle collision. 


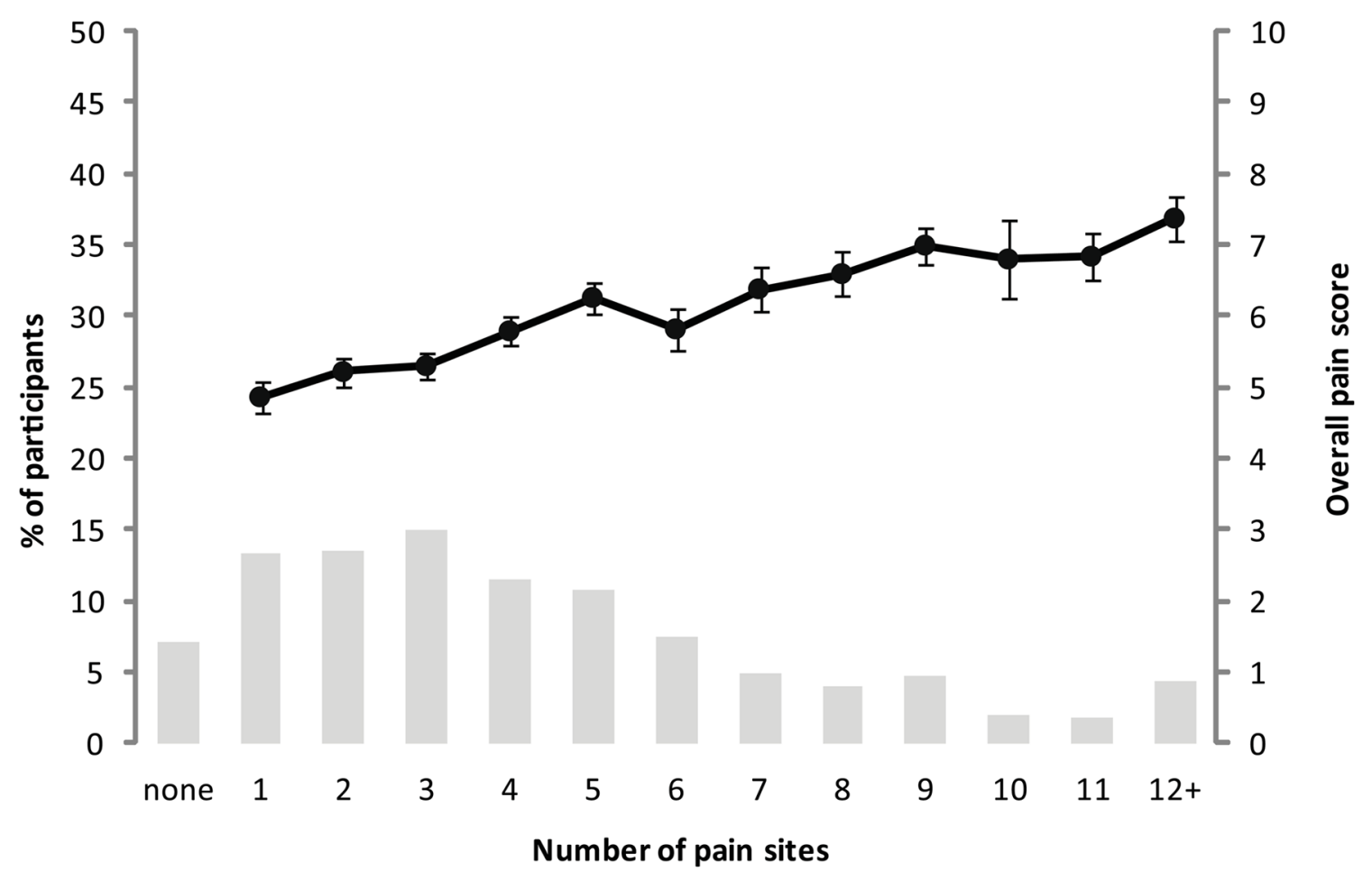

Figure 2.

Percentage of participants and overall pain scores (mean \pm standard error) by number of pain sites in the immediate aftermath of motor vehicle collision. Number of pain sites and overall pain score were significantly associated (Pearson $r=0.38, p<0.001$ ). 


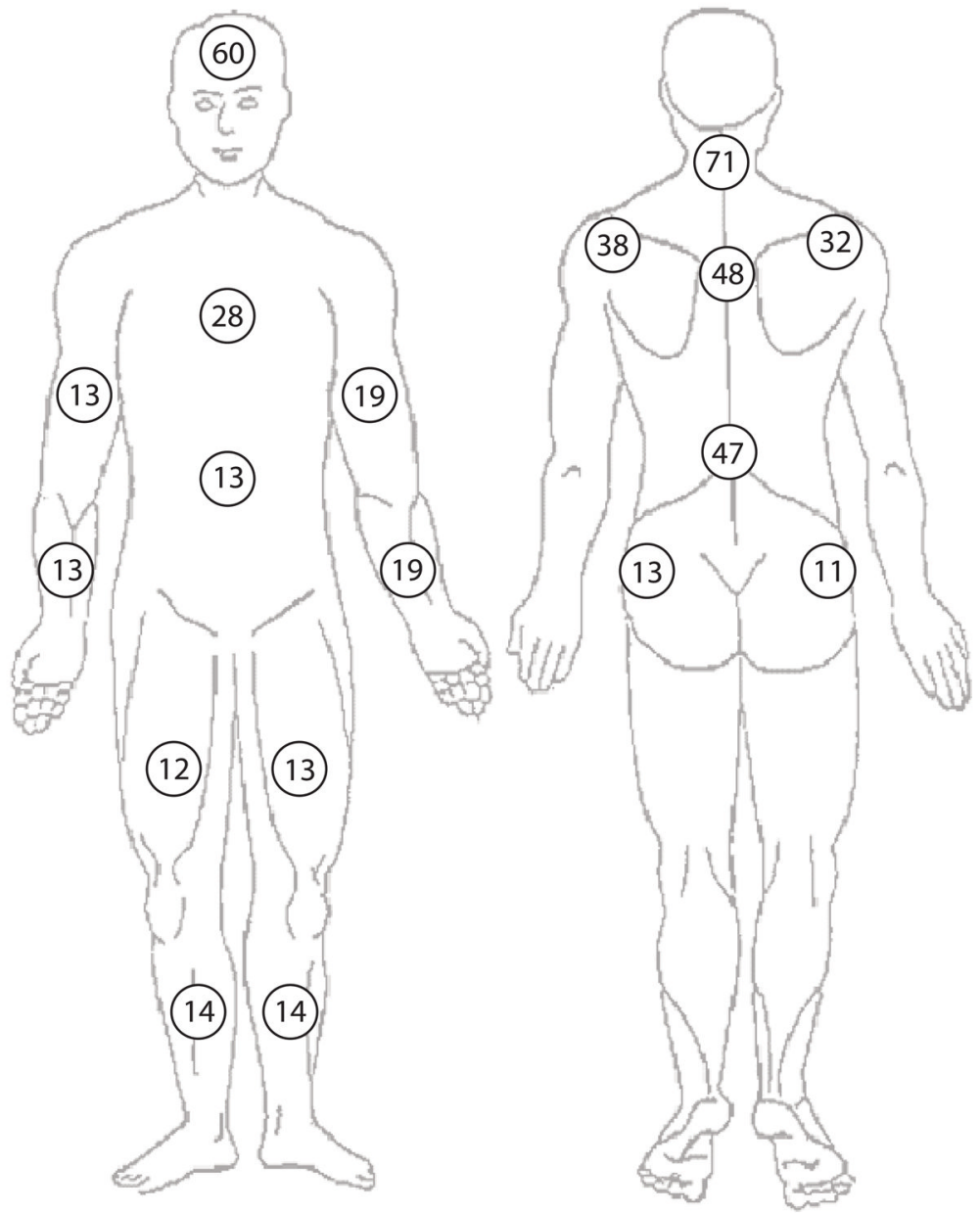

Figure 3.

Percentage of participants reporting pain in the early aftermath of motor vehicle collision according to body region (pain score $>0,0-10$ scale). 


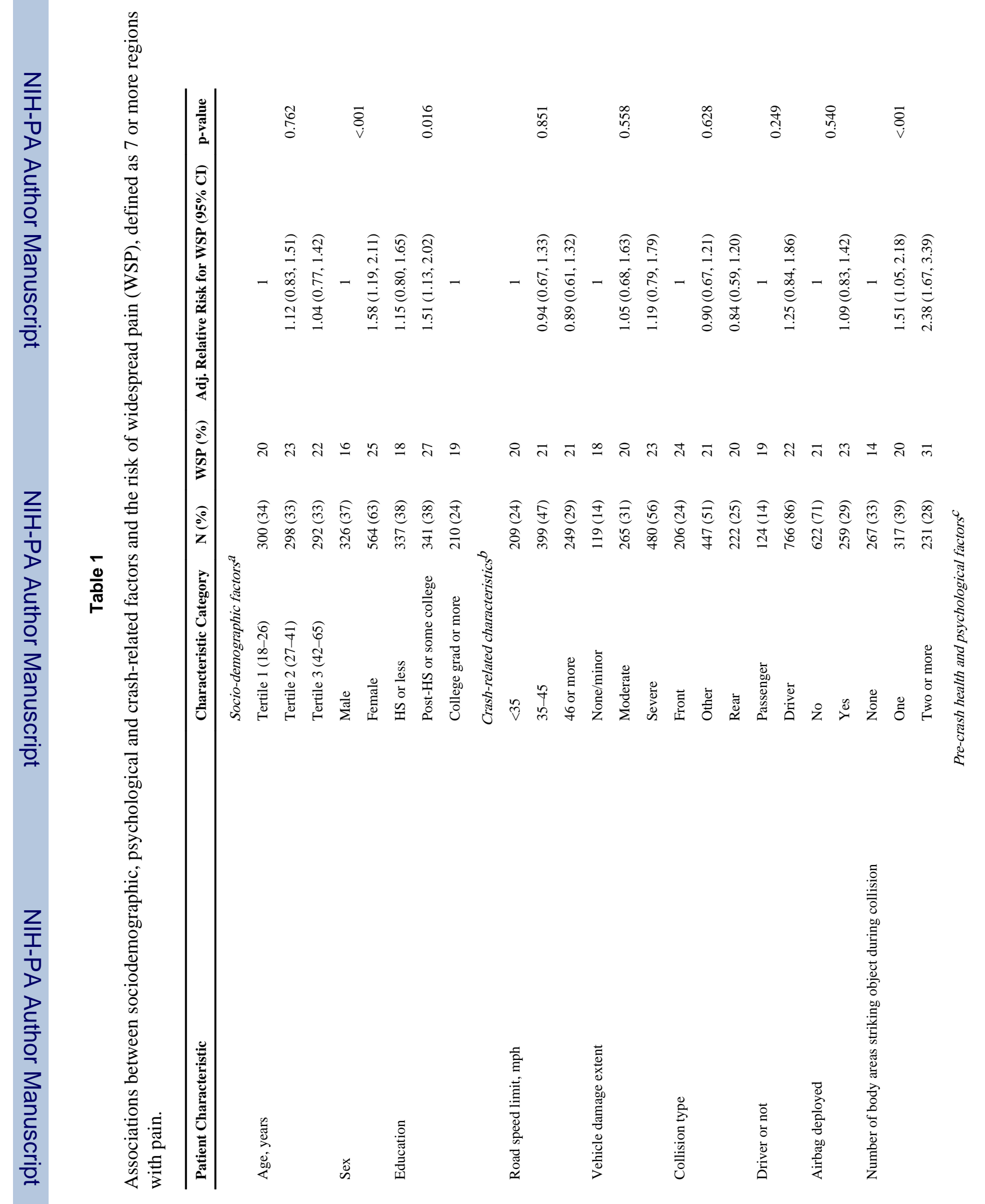


Bortsov et al.

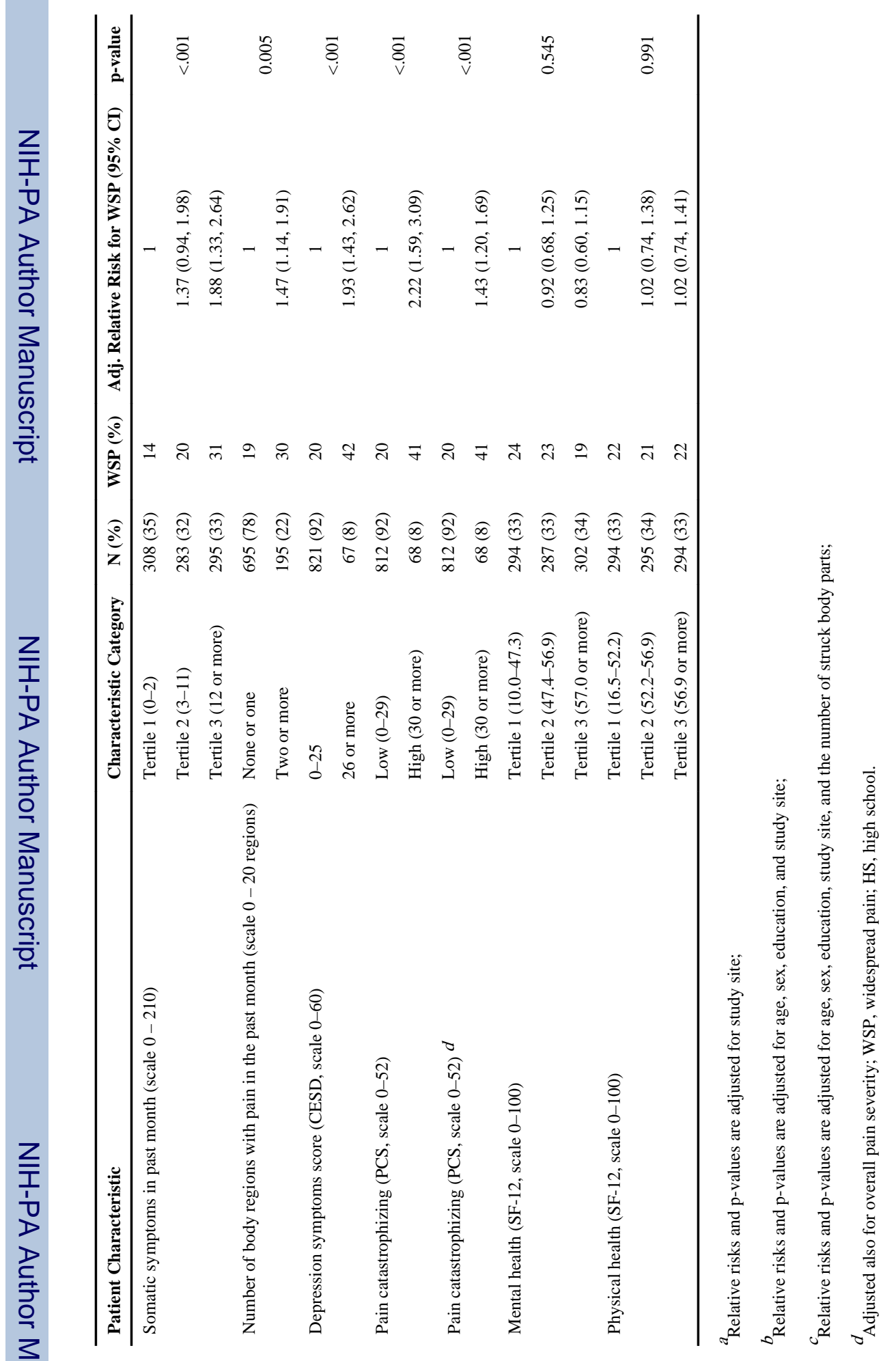


